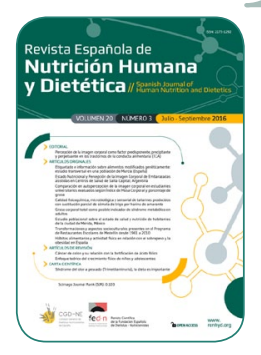

ORIGINAL

\title{
Hábitos alimentarios y actividad física en relación con el sobrepeso y la obesidad en España
}

\author{
María Marqueta de Salas $^{a, b, *}$, José Javier Martín-Ramiro ${ }^{a, b}$, Lorena Rodríguez Gómez ${ }^{a, b}$, \\ Diego Enjuto Martínez ${ }^{a}$, José Juan Juárez Soto ${ }^{c}$
}

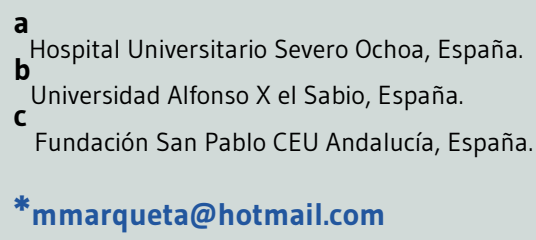

Recibido el 9 de mayo de 2016; aceptado el 26 de agosto de 2016.

Hábitos alimentarios y actividad física en relación con el sobrepeso y la obesidad en España

\section{PALABRAS CLAVE}

Obesidad;

Sobrepeso;

Hábitos Alimentarios;

Ejercicio Físico;

Encuestas Nacionales de Salud;

Encuestas y Cuestionarios.

\section{RESUMEN}

Introducción: El objetivo del presente estudio es analizar los hábitos de alimentación y ejercicio físico de la población española en el año 2012 y su relación con el sobrepeso y la obesidad.

Material y Métodos: Estudio transversal de la Encuesta Nacional de Salud de España del año 2012. Se realizó un análisis descriptivo de los hábitos alimentarios y actividad física en la población general y por sexos con edades comprendidas entre los 18 y los 90 años y un análisis de regresión logística multinomial calculando las RRR crudas y ajustadas a una serie de variables sociodemográficas para valorar el riesgo de presentar sobrepeso y obesidad según la adherencia a las recomendaciones nutricionales dadas por la Sociedad Española de Nutrición Comunitaria (SENC), el consumo o no de alcohol y la realización de actividad física regular.

Resultados: Menos del 50\% de la población española siguió las recomendaciones dadas por la SENC en el consumo de pasta y arroz, verduras, embutidos y dulces. Las mujeres siguieron más las recomendaciones de frutas, verduras, lácteos, pescado, embutidos, refrescos, comida rápida y aperitivos y los hombres las de pasta y arroz, pan y cereales y legumbres, en ambos casos con significación estadística. Más de la mitad de los encuestados $(81,4 \%)$ no realizó actividad física durante su tiempo libre siendo los hombres los que la realizaban con mayor frecuencia. El consumo diario de fruta se asoció a un mayor riesgo de presentar sobrepeso (RRR ajustada=0,77; $\mathrm{p}=, 008)$ y el consumo ocasional de dulces frente al diario se asoció a un mayor riesgo de tener sobrepeso (RRR ajustada $=0,83 ; p=, 005$ ) y obesidad (RRR ajustada $=0,73 ; p<, 001$ ). La realización de actividad física varias veces en semana está directamente relacionada con la disminución significativa del exceso de peso $(p<, 001)$.

Conclusiones: La población española realiza poca actividad física en su tiempo libre. La asociación de determinados hábitos alimentarios y la presencia de exceso de peso no está clara, y este trabajo se suma a las contradicciones encontradas en la literatura al respecto. 
Food habits and physical activity in relation to overweight and obesity in Spain

\section{KEYWORDS}

Obesity;

Overweight;

Food Habits;

Motor Activity;

National Health

Surveys;

Surveys and

Questionnaires.

\section{ABSTRACT}

Introduction: The aim of this paper is to determine the eating habits and the physical activity in the Spanish population along 2012 and to analyze its relationship with overweight and obesity.

Material and Methods: Cross-sectional study of the National Health Survey in 2012. A descriptive analysis of the eating habits and the physical activity was conducted within the general population and also within the genders for ages between 18 and 90 of age. Also, a multinomial logistic regression analysis was performed calculating the RRR adjusted to a series of sociodemographic variables. The goal was to assess the risk of overweight and obesity and know whether the individuals followed or not the recommendations given by the Spanish Society of Community Nutrition (SENC) in relation to eating habits, alcohol consumption and regular physical activity.

Results: Less than $50 \%$ of the Spanish population followed the recommendations given by the SENC in the consumption of pasta and rice, vegetables, sausages and sweets. Women significantly followed the recommendations of taking fruits, vegetables, dairy products, fish, sausages, soft drinks, fast food and snacks and men followed the recommendations of pasta and rice, bread and cereals and legumes, in a statistically significant manner in both cases. More than a half of those surveyed (81.4\%) did not physical activity during their free time being men the ones who performed physical activity more frequently. Daily consumption of fruit was associated with a higher risk of overweight (RRR adjusted $=0.77 ; p=.008$ ) and occasional consumption of sweets compared to the daily one showed a higher risk of overweight (RRR adjusted $=0.83 ; p=.005$ ) and obesity (RRR adjusted $=0.73 ; p<.001$ ). Physical activity several times a week or monthly decreased the latter risk significantly $(p<.001)$.

Conclusions: The Spanish population does not exercise enough in its free time. Association between eating habits and overweight and obesity is not clear and this paper follows the lead of the contradictions found in literature.

\section{CITA}

Marqueta de Salas M, Martín-Ramiro JJ, Rodríguez Gómez L, Enjuto Martínez D, Juárez Soto JJ. Hábitos alimentarios y actividad física en relación con el sobrepeso y la obesidad en España. Rev Esp Nutr Hum Diet. 2016; 20(3): 224235. doi: 10.14306/renhyd.20.3.237

\section{INTRODUCCIÓN}

La obesidad es un importante problema de salud pública a nivel mundial y su prevalencia está aumentando de forma alarmante. Según un informe elaborado por la Organización Mundial de la Salud (OMS), en el año 2008 había en el mundo 310 millones de obesos y 1.700 millones de personas con sobrepeso ${ }^{1}$. En 2014 esas cifras alcanzaron los 600 millones de personas en el caso de la obesidad y 1.900 millones para el caso del sobrepeso². En Europa la tendencia ha sido similar, observando niveles crecientes en la prevalencia de obesidad en los diferentes países en el período comprendido entre 1975 y 20053. En España, la prevalencia de obesidad también ha ido en aumento en las últimas décadas. En 1987, año en el que se publicó la primera Encuesta Nacional de Salud en España (ENS), la prevalencia de obesidad en adultos fue del $7,7 \%$. Esa cifra se duplicó en 2001 alcanzando el 13,6\% ${ }^{4}$ y en 2006 se situaba en el $15,6 \%$ según datos obtenidos del Instituto Nacional de Estadística a partir de la ENS de ese mismo año.

Entre las principales causas de sobrepeso y obesidad se encuentra el desequilibrio entre la ingesta y el gasto energético. En los últimos años en España se han realizado varios estudios con el objetivo de describir los hábitos alimentarios de la población española. En 2011 se realizó la Encuesta Nacional de Ingesta Dietética ${ }^{5}$ (ENIDE) en la que se evaluó la 
ingesta alimentaria de forma directa en todo el territorio nacional. Las conclusiones obtenidas de este trabajo mostraron que la población española consumía menos fruta, verdura, legumbres y frutos secos de lo recomendado y más carne grasa, embutido y bollería. En 2015 se publicó un consenso sobre la metodología de las encuestas de hábitos alimentarios, estudios de nutrición, ejercicio físico y otros estilos de vida $^{6}$, y en este mismo año se publicó también el estudio ANIBES7, que tuvo en cuenta datos antropométricos, de ingesta y balance energético, concluyendo que los principales grupos de alimentos consumidos por la población fueron los cereales, carne y embutidos, aceite, leche y derivados.

Con respecto a la actividad física, diversos trabajos hacen referencia a la importancia de su relación con la presencia de sobrepeso y obesidad. En 2013 se publicó un documento de consenso en el que se relacionó el sedentarismo y la inactividad física con el sobrepeso y la obesidad ${ }^{8}$.

En las últimas cuatro encuestas nacionales de salud, 2001, 2003, 2006 y 2012, se han incluido varias preguntas en relación a la frecuencia de consumo de ciertos grupos de alimentos. El gran tamaño muestral de la ENS hace de ella una herramienta muy útil para poder estudiar los hábitos alimentarios y la actividad física de la población general.

El objetivo de este trabajo es analizar los hábitos alimentarios y actividad física de la población adulta española en el año 2012 y su relación con la presencia de sobrepeso y obesidad.

\section{MATERIAL Y MÉTODOS}

\section{Diseño y Población de estudio}

Se trata de un estudio transversal realizado a partir de la Encuesta Nacional de Salud del año $2012^{9}$ (ENS-2012) que englobó una muestra de 20.069 personas con edades comprendidas entre 18 y 90 años, con una media de edad de 52,15 años y una desviación estándar de 18,16; de los cuales 9.315 fueron hombres $(46,4 \%)$ y 10.754 mujeres $(53,6 \%)$.

\section{Descripción de Variables}

Las personas seleccionadas en este estudio se dividieron en tres categorías considerando sus valores de Índice de Masa Corporal (IMC), siendo éste el método recomendado por la $\mathrm{OMS}^{10}$ para identificar el sobrepeso y la obesidad. Para el cálculo del mismo se aplicó la siguiente fórmula, siendo los valores de peso y talla autorreferidos por los encuestados:

$$
\text { IMC = Peso }(\mathrm{kg}) / \text { Talla }\left(\mathrm{m}^{2}\right)
$$

Los datos obtenidos se agruparon de la siguiente manera: Normopeso (IMC:18,5-24,9), Sobrepeso (IMC:25-29,9), Obesidad (IMC $\geq 30$ ), y se excluyeron las personas con bajo peso (IMC $<18,5)$.

La población del estudio, extraída de la ENS-2012, estuvo configurada por las personas que respondieron a las siguientes preguntas:

- Pregunta 133: ¿con qué frecuencia consume los siguientes alimentos? (fruta, pasta y arroz, pan y cereales, verduras, lácteos, huevos, carne, pescado, legumbre, embutidos, dulces, refrescos, comida rápida y aperitivos).

Las opciones de respuesta fueron: 1 , diario; 2 , tres o más veces en semana pero no diario; 3 , una o dos veces a la semana; 4, menos de una vez a la semana; y 5, nunca o casi nunca. La Sociedad Española de Nutrición Comunitaria (SENC) ${ }^{11}$ recomienda: consumo diario de fruta, pasta y arroz, pan y cereales, verdura y lácteos; consumo semanal de huevos, carne, pescado y legumbres; y consumo ocasional de embutido, dulces, refrescos, comida rápida y aperitivos. Tomando como referencia estas recomendaciones, los resultados obtenidos de la ENS-2012 se agruparon como:

- Consumo diario: incluyó la categoría 1

- Consumo semanal: incluyó las categorías 2 y 3

- Consumo ocasional: incluyó las categorías 4 y 5

- Pregunta 129: ¿cuál de estas probabilidades describe mejor la frecuencia con la que realiza alguna actividad física en su tiempo libre?

Las opciones de respuesta eran: 1, nunca; 2, ocasional; 3 , varias veces al mes; y 4 , varias veces a la semana. Para la realización del estudio estas opciones se estructuraron del siguiente modo:

- Actividad física ocasional: incluyó las categorías 1 y 2

- Actividad física mensual: incluyó la categoría 3

- Actividad física semanal: incluyó la categoría 4

- Pregunta 124: consumo de cerveza y vino en una semana durante los últimos 12 meses.

Las respuestas se agruparon en sí o no.

\section{Análisis Estadístico}

El análisis estadístico se realizó mediante el programa SPSS 23.0. En primer lugar, se realizó un análisis descriptivo acerca de los hábitos alimentarios de la población española y se comprobó si las recomendaciones referentes al consumo de los grupos de alimentos de la SENC se siguieron más de lo que cabía esperar en hombres o mujeres. Se contrastaron, mediante 
el estadístico Chi Cuadrado $\left(\chi^{2}\right)$, las relaciones bivariadas del sexo con el consumo de cada grupo de alimentos, alcohol (cerveza y vino) y la actividad física en momentos de ocio. Dado el elevado tamaño de la muestra que pudo provocar significación estadística cuando las diferencias encontradas en la práctica pudieran ser insignificantes, se siguieron las recomendaciones de Cohen, por lo que se consideró el coeficiente de correlación V de Cramér como índice de tamaño de efecto, considerándose pequeño: entre ,10 y ,29; mediano: entre ,30 y ,49; o grande: $\geq$,50. En caso de significación y tamaño de efecto grande, se estimaron los residuales tipificados corregidos de Haberman $\left(A_{\mathrm{ij}}\right)$, con objeto de detallar si el porcentaje de hombres y mujeres que siguieron las recomendaciones de consumo de cada grupo de alimento fue mayor o menor al esperado por azar o bajo la condición de independencia. Como para cualquier puntuación tipificada, dichos residuos se consideraron estadísticamente significativos a partir de un valor superior a $1,96(\alpha=, 05)$ o mayor a $2,58(\alpha=, 01)$.

Se realizó un análisis de regresión logística multinomial para estimar las tasas de posibilidad de riesgo (RRR o razón de riesgo relativo) de obesidad y sobrepeso frente al normopeso (categoría de referencia) según los consumos de diferentes grupos de alimentos, alcohol (cerveza y vino) y la frecuencia de la actividad física durante el ocio. Las catego- rías tomadas como referencia fueron las recomendaciones de la SENC para cada grupo de alimentos, el no consumo de cerveza y vino, y la no realización de actividad física durante el ocio. Se calcularon las RRR crudas y las ajustadas a las variables sociodemográficas (edad, sexo, estado civil, clase social, comunidad autónoma y tamaño del municipio) que resultaron significativas en un estudio previo titulado características sociodemográficas como factores de riesgo para la obesidad y el sobrepeso en la población adulta española ${ }^{12}$. Siguiendo las recomendaciones de Cohen, se consideraron los tamaños de efecto en función del valor de la RRR: grande: $\geq 9$; mediano: 3,45-8,99; y pequeño: 1,49-3,44.

\section{RESULTADOS}

La prevalencia de sobrepeso en la población adulta española en el año 2012 fue del 39,0\% (47,3\% en hombres y 31,1\% en mujeres) y la de obesidad del $18,5 \%$ (19,3\% en hombres y $17,8 \%$ en mujeres).

La Tabla 1 muestra las frecuencias de consumo de cada grupo de alimentos y la actividad física realizada en el total de la población y por sexos. El consumo recomendado de fruta,

Tabla 1. Distribución de la población total y por sexo a partir de la Encuesta Nacional de Salud española (2012) ${ }^{9}$, según consumo de grupos de alimentos recomendado por la Sociedad Española de Nutrición Comunitaria (SENC), consumo de vino y cerveza y actividad física durante el ocio.

\begin{tabular}{|c|c|c|c|c|c|c|c|}
\hline & \multicolumn{2}{|r|}{ Total } & \multicolumn{2}{|r|}{ Hombre } & \multicolumn{2}{|r|}{ Mujer } \\
\hline & & n & \% (IC 95\%) & $\mathrm{n}$ & \% (IC 95\%) & $\mathrm{n}$ & \% (IC 95\%) \\
\hline \multirow{15}{*}{ 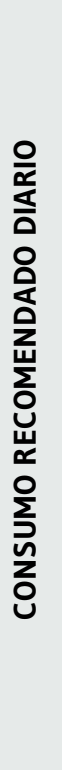 } & D & 13211 & $65,9(65,2-66,6)$ & 5662 & $60,9(59,9-61,9)$ & 7549 & $70,3(69,4-71,1)$ \\
\hline & $\mathbf{S}$ & 5391 & $26,9(26,3-27,5)$ & 2821 & $30,3(29,4-31,3)$ & 2570 & $23,9(23,1-24,7)$ \\
\hline & 0 & 1442 & $7,2(6,8-7,5)$ & 819 & $8,8(8,2-9,4)$ & 623 & $5,8(5,3-6,2)$ \\
\hline & D & 2632 & $13,1(12,7-13,6)$ & 1339 & $14,4(13,7-15,1)$ & 1293 & $12,0(11,4-12,6)$ \\
\hline & $\mathbf{S}$ & 16582 & $82,7(82,2-83,3)$ & 7649 & $82,2(81,4-83,0)$ & 8933 & $83,2(82,5-83,9)$ \\
\hline & 0 & 827 & $4,1(3,8-4,4)$ & 313 & $3,4(3,0-3,7)$ & 514 & $4,8(4,4-5,2)$ \\
\hline & D & 16854 & $84,1(83,6-84,6)$ & 7998 & $86,0(85,3-86,7)$ & 8856 & $82,5(81,7-83,2)$ \\
\hline & $\mathbf{S}$ & 2477 & $12,4(11,9-12,8)$ & 1035 & $11,1(10,5-11,8)$ & 1442 & $13,4(12,8-14,1)$ \\
\hline & 0 & 711 & $3,5(3,3-3,8)$ & 268 & $2,9(2,5-3,2)$ & 443 & $4,1(3,7-4,5)$ \\
\hline & D & 9665 & $48,2(47,5-48,9)$ & 3956 & $42,5(41,5-43,5)$ & 5709 & $53,2(52,2-54,1)$ \\
\hline & $\mathbf{S}$ & 9451 & $47,2(46,5-47,8)$ & 4766 & $51,3(50,2-52,3)$ & 4685 & $43,6(42,7-44,6)$ \\
\hline & 0 & 923 & $4,6(4,3-4,9)$ & 577 & $6,2(5,7-6,7)$ & 346 & $3,2(2,9-3,6)$ \\
\hline & D & 17169 & $85,7(85,2-86,2)$ & 7766 & $83,5(82,7-84,3)$ & 9403 & $87,6(86,9-88,2)$ \\
\hline & $\mathbf{S}$ & 2002 & $10,0(9,6-10,4)$ & 1083 & $11,6(11,0-12,3)$ & 919 & $8,6(8,0-9,1)$ \\
\hline & 0 & 868 & $4,3(4,0-4,6)$ & 452 & $4,9(4,4-5,3)$ & 416 & $3,9(3,5-4,2)$ \\
\hline
\end{tabular}




\begin{tabular}{|c|c|c|c|c|c|c|c|c|}
\hline & \multicolumn{2}{|r|}{ Total } & \multicolumn{2}{|r|}{ Hombre } & \multicolumn{2}{|r|}{ Mujer } \\
\hline & & & n & $\%$ (IC 95\%) & n & $\%$ (IC 95\%) & $\mathrm{n}$ & $\%$ (IC 95\%) \\
\hline & \multirow{3}{*}{ HU } & D & 249 & $1,2(1,1-1,4)$ & 157 & $1,7(1,4-1,9)$ & 92 & $0,9(0,7-1,0)$ \\
\hline \multirow{11}{*}{ 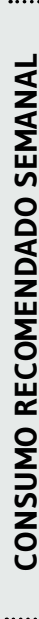 } & & $\mathbf{S}$ & 17022 & $84,9(84,4-85,4)$ & 7939 & $85,4(84,6-86,1)$ & 9083 & $84,6(83,9-85,3)$ \\
\hline & & 0 & 2769 & $13,8(13,3-14,3)$ & 1205 & $13,0(12,3-13,6)$ & 1564 & $14,6(13,9-15,2)$ \\
\hline & \multirow{3}{*}{ CA } & D & 1772 & $8,8(8,4-9,2)$ & 986 & $10,6(10,0-11,2)$ & 786 & $7,3(6,8-7,8)$ \\
\hline & & $\mathbf{s}$ & 17310 & $86,4(85,9-86,8)$ & 8012 & $86,2(85,4-86,8)$ & 9298 & $86,6(85,9-87,2)$ \\
\hline & & 0 & 956 & $4,8(4,4-5,1)$ & 302 & $3,2(2,9-3,6)$ & 654 & $6,1(5,6-6,5)$ \\
\hline & \multirow{3}{*}{ PE } & D & 474 & $2,4(2,1-2,6)$ & 206 & $2,2(1,9-2,5)$ & 268 & $2,5(2,2-2,8)$ \\
\hline & & S & 17522 & $87,4(87,0-87,9)$ & 8034 & $86,4(85,7-87,1)$ & 9488 & $88,4(87,7-89,0)$ \\
\hline & & 0 & 2042 & $10,2(9,8-10,6)$ & 1059 & $11,4(10,7-12,0)$ & 983 & $9,2(8,6-9,7)$ \\
\hline & \multirow{3}{*}{ LE } & D & 265 & $1,3(1,2-1,5)$ & 117 & $1,3(1,0-1,5)$ & 148 & $1,4(1,1-1,6)$ \\
\hline & & $\mathbf{S}$ & 16689 & $83,3(82,8-83,8)$ & 7868 & $84,6(83,9-85,3)$ & 8821 & $82,1(81,4-82,9)$ \\
\hline & & 0 & 3084 & $15,4(14,9-15,9)$ & 1313 & $14,1(13,4-14,8)$ & 1771 & $16,5(15,8-17,2)$ \\
\hline \multirow{15}{*}{ 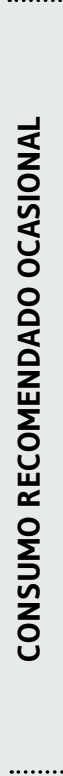 } & \multirow{3}{*}{ EM } & D & 2383 & $11,9(11,4-12,3)$ & 1454 & $15,6(14,9-16,4)$ & 929 & $8,7(8,1-9,2)$ \\
\hline & & S & 9660 & $48,2(47,5-48,9)$ & 4852 & $52,2(51,2-53,2)$ & 4808 & $44,8(43,9-45,7)$ \\
\hline & & 0 & 7986 & $39,9(39,2-40,5)$ & 2992 & $32,2(31,2-33,1)$ & 4994 & $46,5(45,6-47,5)$ \\
\hline & \multirow{3}{*}{ DU } & D & 5826 & $29,1(28,5-29,7)$ & 2684 & $28,9(27,9-29,8)$ & 3142 & $29,3(28,4-30,2)$ \\
\hline & & S & 6158 & $30,8(30,1-31,4)$ & 2858 & $30,7(29,8-31,7)$ & 3300 & $30,8(29,9-31,6)$ \\
\hline & & 0 & 8036 & $40,1(39,5-40,8)$ & 3753 & $40,4(39,4-41,4)$ & 4283 & $39,9(39,0-40,9)$ \\
\hline & \multirow{3}{*}{ RE } & D & 1995 & $10,0(9,5-10,4)$ & 1177 & $12,7(12,0-13,3)$ & 818 & $7,6(7,1-8,1)$ \\
\hline & & $\mathbf{S}$ & 4294 & $21,5(20,9-22,0)$ & 2260 & $24,3(23,4-25,2)$ & 2034 & $19,0(18,2-19,7)$ \\
\hline & & 0 & 13717 & $68,6(67,9-69,2)$ & 5852 & $63,0(62,0-64,0)$ & 7865 & $73,4(72,5-74,2)$ \\
\hline & \multirow{3}{*}{ RA } & D & 282 & $1,4(1,2-1,6)$ & 203 & $2,2(1,9-2,5)$ & 79 & $0,7(0,6-0,9)$ \\
\hline & & $\mathbf{S}$ & 3912 & $19,6(19,0-20,1)$ & 2155 & $23,2(22,3-24,1)$ & 1757 & $16,4(15,7-17,1)$ \\
\hline & & 0 & 15815 & $79,0(78,5-79,6)$ & 6932 & $74,6(73,7-75,5)$ & 8883 & $82,9(82,1-83,6)$ \\
\hline & \multirow{3}{*}{ AP } & D & 287 & $1,4(1,3-1,6)$ & 176 & $1,9(1,6-2,2)$ & 111 & $1,0(0,8-1,2)$ \\
\hline & & S & 4283 & $21,4(20,8-22,0)$ & 2226 & $24,0(23,1-24,8)$ & 2057 & $19,2(18,4-19,9)$ \\
\hline & & 0 & 15445 & $77,2(76,6-77,7)$ & 6891 & $74,2(73,2-75,0)$ & 8554 & $79,8(79,0-80,5)$ \\
\hline \multirow{7}{*}{ 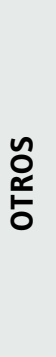 } & \multirow{2}{*}{ CE } & sí & 5818 & $63,8(62,8-64,8)$ & 3947 & $67,8(66,6-69,0)$ & 1871 & $56,6(54,9-58,3)$ \\
\hline & & NO & 3305 & $36,2(35,2-37,2)$ & 1871 & $32,2(30,9-33,4)$ & 1434 & $43,4(41,7-45,1)$ \\
\hline & \multirow{2}{*}{ VI } & sí & 5211 & $57,0(56,0-58,0)$ & 3267 & $56,0(54,7-57,3)$ & 1944 & $58,7(57,0-60,4)$ \\
\hline & & NO & 3934 & $43,0(42,0-44,0)$ & 2568 & $44,0(42,7-45,3)$ & 1366 & $41,3(39,6-43,0)$ \\
\hline & \multirow{3}{*}{ AFO } & $\mathbf{S}$ & 1557 & $7,8(7,4-8,1)$ & 952 & $10,2(9,6-10,8)$ & 605 & $5,6(5,2-6,1)$ \\
\hline & & M & 2170 & $10,8(10,4-11,2)$ & 1392 & $15,0(14,2-15,7)$ & 778 & $7,2(6,7-7,7)$ \\
\hline & & 0 & 16326 & $81,4(80,9-81,9)$ & 6962 & $74,8(73,9-75,7)$ & 9364 & $87,1(86,5-87,8)$ \\
\hline
\end{tabular}

FR: fruta; PA: pasta y arroz; PC: pan y cereales; VE: verduras; LA: lácteos; HU: huevos; CA: carne; PE: pescado; LE: legumbres; EM: embutidos; DU: dulces; RE: refrescos; RA: comida rápida; AP: aperitivos; CE: cerveza; VI: vino; AFO: actividad física durante el ocio.

D: diario; S: semanal; O: ocasional; M: mensual.

\%: Porcentaje dentro del sexo. 
pasta y verduras es diario y sin embargo el $26,9 \%, 82,7 \%$ y $47,2 \%$ respectivamente de la población no lo realiza diariamente sino semanal. En cuanto a los grupos de alimentos con consumos recomendados ocasionales cabe destacar que el $48,2 \%$ de la población realiza un consumo semanal de embutidos, el $30,8 \%$ de dulces, el $21,5 \%$ de refrescos, el $21,4 \%$ de comida rápida y el $21,4 \%$ de aperitivos.

En cuanto a la relación entre el sexo y el consumo de los diferentes grupos de alimentos, se detectó un porcentaje de mujeres significativamente mayor al esperado por azar en el consumo diario de frutas $(A R=14,0)$, verduras $(A R=15,0)$ y lácteos $(A R=8,2)$; mientras que en hombres, dicho porcentaje fue significativamente superior para el de pasta $(A R=4,9)$ y pan-cereales $(A R=6,8)$. También fueron significativas $(p<, 001)$ todas las relaciones entre el sexo y los grupos de alimentos cuyo consumo recomendado era de varias veces a la semana, aunque todos los tamaños de efecto fueron muy pequeños. Se encontraron más mujeres de las que cabía esperar que tomaron semanalmente pescado ( $A R=4,9)$, y más hombres que tomaron legumbres $(A R=4,7)$. Tanto hombres como mujeres presentaron un consumo recomendado o semanal de carne y huevos similar al esperado por azar $(A R<1,96)$, aunque el porcentaje de ingesta diaria (no recomendada) de carne $(A R=8,2)$ y huevos $(A R=5,3)$ fue significativamente mayor al esperado en los hombres. Respecto al grupo de alimentos cuyo consumo recomendado era ocasional, no se relacionó el sexo con el consumo de dulces, aunque se detectaron significaciones con tamaños de efecto pequeños con el resto de alimentos. Destacó que el porcentaje de mujeres que ingirieron ocasionalmente embutidos $(A R=20,7)$, refrescos $(A R=15,3)$, comida rápida $(A R=14,3)$ y aperitivos $(A R=9,5)$ fue significativamente mayor al esperado bajo la condición de independencia entre las variables. Respecto al alcohol, se encontró un consumo de cerveza mayor al esperado $(A R=10,7)$ en hombres, y de vino $(A R=2,5)$ en mujeres. Por último, se detectó una relación significativa entre sexo y actividad física durante el ocio $(p<, 001)$, con un tamaño de efecto pequeño. Resultó que el porcentaje de mujeres sin actividad física fue superior al esperado por azar $(A R=22,4)$, mientras que más hombres de los que cabía esperar la realizaban de forma mensual $(A R=17,5)$ o semanal $(\mathrm{AR}=12,1)$.

La Tabla 2 muestra las frecuencias de consumo de cada grupo de alimentos y la actividad física realizada según el grado de obesidad. Con respecto a la ingesta de fruta, la mayoría de personas con obesidad y sobrepeso tomaron fruta

Tabla 2. Distribución de la población con normopeso, sobrepeso y obesidad a partir de la Encuesta Nacional de Salud española $(2012)^{9}$, según el consumo de cada grupo de alimentos siguiendo las recomendaciones dadas por la Sociedad Española de Nutrición Comunitaria (SENC), consumo de vino y cerveza y realización de actividad física durante el ocio.

\begin{tabular}{|c|c|c|c|c|c|c|c|c|}
\hline & \multicolumn{2}{|c|}{ Normopeso } & \multicolumn{2}{|c|}{ Sobrepeso } & \multicolumn{2}{|c|}{ Obesidad } \\
\hline & & & $\mathrm{n}$ & \% (IC 95\%) & n & \% (IC 95\%) & $\mathrm{n}$ & \% (IC 95\%) \\
\hline \multirow{15}{*}{ 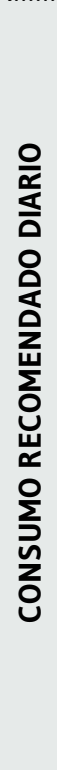 } & \multirow{3}{*}{ FR } & D & 4889 & $41,0(40,1-41,9)$ & 4806 & $40,3(39,4-41,2)$ & 2226 & $18,7(18,0-19,4)$ \\
\hline & & $\mathbf{S}$ & 2297 & $46,3(44,9-47,7)$ & 1797 & $36,2(34,9-37,6)$ & 867 & $17,5(16,4-18,5)$ \\
\hline & & 0 & 647 & $48,3(45,6-51,0)$ & 460 & $34,4(31,8-36,9)$ & 232 & $17,3(15,3-19,4)$ \\
\hline & \multirow{3}{*}{ PA } & D & 1050 & $44,8(42,7-46,8)$ & 874 & $37,3(35,3-39,2)$ & 421 & $18,0(16,4-19,5)$ \\
\hline & & $\mathbf{S}$ & 6496 & $43,0(42,2-43,8)$ & 5874 & $38,9(38,1-39,7)$ & 2738 & $18,1(17,5-18,7)$ \\
\hline & & 0 & 287 & $37,4(33,9-40,9)$ & 314 & $40,9(37,4-44,5)$ & 166 & $21,6(18,7-24,6)$ \\
\hline & \multirow{3}{*}{ PC } & D & 6523 & $42,7(41,9-43,5)$ & 5974 & $39,1(38,3-39,8)$ & 2789 & $18,2(17,6-18,9)$ \\
\hline & & $\mathbf{S}$ & 1027 & $45,0(43,0-47,1)$ & 853 & $37,4(35,4-39,4)$ & 401 & $17,6(16,0-19,2)$ \\
\hline & & 0 & 283 & $43,3(39,4-47,1)$ & 236 & $36,1(32,3-39,8)$ & 135 & $20,6(17,5-23,8)$ \\
\hline & \multirow{3}{*}{ VE } & D & 3801 & $43,0(41,9-44,0)$ & 3458 & $39,1(38,1-40,1)$ & 1588 & $17,9(17,1-18,5)$ \\
\hline & & S & 3662 & $42,9(41,8-43,9)$ & 3288 & $38,5(37,4-39,5)$ & 1592 & $18,6(17,8-19,5)$ \\
\hline & & 0 & 371 & $44,7(41,2-48,1)$ & 315 & $38,0(34,6-41,3)$ & 144 & $17,3(14,7-20,0)$ \\
\hline & \multirow{3}{*}{ LA } & D & 6687 & $42,9(42,1-43,7)$ & 6070 & $38,9(38,2-39,7)$ & 2832 & $18,2(17,6-18,8)$ \\
\hline & & $\mathbf{S}$ & 802 & $43,6(41,3-45,9)$ & 693 & $37,7(35,5-39,9)$ & 343 & $18,7(16,8-20,5)$ \\
\hline & & 0 & 343 & $43,3(39,8-46,8)$ & 299 & $37,8(34,3-41,2)$ & 150 & $18,9(16,1-21,7)$ \\
\hline
\end{tabular}




\begin{tabular}{|c|c|c|c|c|c|c|c|c|}
\hline & \multicolumn{2}{|c|}{ Normopeso } & \multicolumn{2}{|c|}{ Sobrepeso } & \multicolumn{2}{|c|}{ Obesidad } \\
\hline & & & n & $\%$ (IC 95\%) & $\mathrm{n}$ & $\%$ (IC 95\%) & n & $\%$ (IC 95\%) \\
\hline \multirow{12}{*}{ 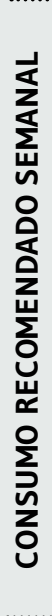 } & \multirow{3}{*}{ HU } & D & 119 & $50,6(44,0-57,2)$ & 74 & $31,5(25,3-37,6)$ & 42 & $17,9(12,8-23,0)$ \\
\hline & & $\mathbf{S}$ & 6735 & $43,4(42,6-44,1)$ & 6015 & $38,7(37,9-39,5)$ & 2786 & $17,9(17,3-18,5)$ \\
\hline & & 0 & 977 & $39,9(37,9-41,9)$ & 974 & $39,8(37,8-41,7)$ & 497 & $20,3(18,7-21,9)$ \\
\hline & \multirow{3}{*}{ CA } & D & 709 & $43,8(41,4-46,3)$ & 616 & $38,1(35,7-40,5)$ & 292 & $18,1(16,1-20,0)$ \\
\hline & & $\mathbf{S}$ & 6725 & $42,7(41,9-43,4)$ & 6163 & $39,1(38,3-39,9)$ & 2872 & $18,2(17,6-18,8)$ \\
\hline & & 0 & 398 & $47,3(43,9-50,7)$ & 283 & $33,7(30,4-36,9)$ & 160 & $19,0(16,3-21,7)$ \\
\hline & \multirow{3}{*}{ PE } & D & 170 & $39,8(35,0-44,6)$ & 169 & $39,6(34,8-44,3)$ & 88 & $20,6(16,6-24,6)$ \\
\hline & & $\mathbf{S}$ & 6746 & $42,4(41,6-43,2)$ & 6227 & $39,1(38,4-39,9)$ & 2933 & $18,4(17,8-19,0)$ \\
\hline & & 0 & 916 & $48,6(46,3-50,8)$ & 666 & $35,3(33,1-37,5)$ & 304 & $16,1(14,4-17,8)$ \\
\hline & \multirow{3}{*}{ LE } & D & 120 & $50,0(43,5-56,5)$ & 80 & $33,3(27,2-39,5)$ & 40 & $16,7(11,7-21,6)$ \\
\hline & & $\mathbf{S}$ & 6462 & $42,5(41,7-43,3)$ & 5936 & $39,0(38,2-39,8)$ & 2812 & $18,5(17,9-19,1)$ \\
\hline & & 0 & 1251 & $45,2(43,3-47,0)$ & 1046 & $37,8(35,9-39,6)$ & 473 & $17,1(15,6-18,5)$ \\
\hline \multirow{15}{*}{ 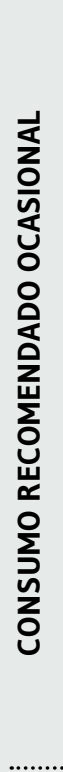 } & \multirow{3}{*}{ EM } & D & 1027 & $45,8(43,7-47,8)$ & 840 & $37,4(35,4-39,4)$ & 377 & $16,8(15,2-18,4)$ \\
\hline & & $\mathbf{s}$ & 3844 & $43,3(42,3-44,4)$ & 3453 & $38,9(37,9-39,9)$ & 1575 & $17,8(16,9-18,5)$ \\
\hline & & 0 & 2959 & $41,7(40,5-42,8)$ & 2765 & $39,0(37,8-40,1)$ & 1372 & $19,3(18,4-20,3)$ \\
\hline & \multirow{3}{*}{ DU } & D & 2485 & $46,8(45,4-48,1)$ & 1935 & $36,4(35,1-37,7)$ & 895 & $16,8(15,8-17,8)$ \\
\hline & & $\mathbf{S}$ & 2525 & $44,8(43,4-46,1)$ & 2128 & $37,7(36,4-39,0)$ & 989 & $17,5(16,5-18,5)$ \\
\hline & & 0 & 2818 & $38,9(37,7-40,0)$ & 2993 & $41,3(40,1-42,4)$ & 1438 & $19,8(18,9-20,8)$ \\
\hline & \multirow{3}{*}{ RE } & D & 899 & $47,7(45,4-50,0)$ & 648 & $34,4(32,2-36,6)$ & 337 & $17,9(16,1-19,6)$ \\
\hline & & $\mathbf{S}$ & 1885 & $46,9(45,3-48,4)$ & 1499 & $37,3(35,8-38,8)$ & 635 & $15,8(14,7-16,9)$ \\
\hline & & 0 & 5040 & $41,0(40,1-41,9)$ & 4905 & $39,9(39,0-40,8)$ & 2351 & $19,1(18,4-19,8)$ \\
\hline & \multirow{3}{*}{ RA } & D & 126 & $46,7(40,5-52,8)$ & 88 & $32,6(26,8-38,4)$ & 56 & $20,7(15,7-25,8)$ \\
\hline & & $\mathbf{S}$ & 1901 & $51,8(50,1-53,4)$ & 1243 & $33,9(32,3-35,4)$ & 527 & $14,4(13,2-15,5)$ \\
\hline & & 0 & 5795 & $40,6(39,8-41,4)$ & 5723 & $40,1(39,3-40,9)$ & 2740 & $19,2(18,6-19,9)$ \\
\hline & \multirow{3}{*}{ AP } & D & 145 & $52,3(46,3-58,4)$ & 84 & $30,3(24,7-35,9)$ & 48 & $17,3(12,7-22,0)$ \\
\hline & & $\mathbf{S}$ & 1971 & $49,0(47,5-50,6)$ & 1411 & $35,1(33,6-36,6)$ & 637 & $15,8(14,7-17,0)$ \\
\hline & & 0 & 5708 & $41,0(40,2-41,9)$ & 5561 & $40,0(39,2-40,8)$ & 2639 & $19,0(18,3-19,6)$ \\
\hline \multirow{7}{*}{$\begin{array}{l}\text { 号 } \\
\text { 号 }\end{array}$} & \multirow{2}{*}{ CE } & sí & 2482 & $44,5(43,2-45,8)$ & 2263 & $40,6(39,3-41,9)$ & 833 & $14,9(14,0-15,9)$ \\
\hline & & NO & 1207 & $39,8(38,1-41,6)$ & 1277 & $42,1(40,4-43,9)$ & 547 & $18,0(16,7-19,4)$ \\
\hline & \multirow{2}{*}{ VI } & sí & 1866 & $38,4(37,1-39,8)$ & 2167 & $44,6(43,2-46,0)$ & 821 & $16,9(15,8-18,0)$ \\
\hline & & NO & 1828 & $48,4(46,8-50,0)$ & 1382 & $36,6(35,1-38,2)$ & 564 & $14,9(13,8-16,1)$ \\
\hline & \multirow{3}{*}{ AFO } & $\mathbf{S}$ & 828 & $55,6(53,0-58,2)$ & 505 & $33,9(31,5-36,3)$ & 156 & $10,5(8,9-12,1)$ \\
\hline & & M & 1183 & $55,9(53,8-58,0)$ & 752 & $35,5(33,5-37,6)$ & 181 & $8,6(7,3-9,8)$ \\
\hline & & 0 & 5826 & $39,8(39,0-40,6)$ & 5810 & $39,7(38,9-40,5)$ & 2989 & $20,4(19,8-21,1)$ \\
\hline
\end{tabular}

FR: fruta; PA: pasta y arroz; PC: pan y cereales; VE: verduras; LA: lácteos; HU: huevos; CA: carne; PE: pescado; LE: legumbres; EM: embutidos; DU: dulces; RE: refrescos; RA: comida rápida; AP: aperitivos; CE: cerveza; VI: vino; AFO: actividad física durante el ocio. 
a diario ( $18,7 \%$ y $40,3 \%$ respectivamente), mientras que en personas con normopeso, el mayor porcentaje lo hizo ocasionalmente (48,3\%). En cuanto al consumo de verdura, el $39,1 \%$ de las personas con sobrepeso comió verdura a diario, mientras que la mayoría de las personas con normopeso $(44,7 \%)$ lo hizo ocasionalmente y el $18,6 \%$ de las personas con obesidad lo hizo semanalmente. El mayor porcentaje de personas con obesidad y sobrepeso consumió embutidos de manera ocasional (19,3\% y 39,0\% respectivamente), frente a la mayoría de personas con normopeso $(45,8 \%)$ que lo hizo diariamente. En lo referente a la ingesta de dulces, el $46,8 \%$ de personas con normopeso consumió dulces a diario, mientras que la mayoría de las personas con obesidad y sobrepeso lo hicieron de forma ocasional $(19,8 \%$ y $41,3 \%$ respectivamente) y algo similar ocurrió con el consumo de refrescos y aperitivos. En cuanto a la ingesta de comida rápida, el mayor porcentaje de personas con obesidad ingirió comida rápida a diario $(20,7 \%)$. Con respecto a la toma de cerveza y vino, destaca que la mayor parte del consumo de cerveza lo hacen las personas con normopeso, mientras que las personas con sobrepeso y obesidad toman vino en mayor medida. El 55,9\% de personas con normopeso realizan actividad física mensualmente y la mayoría de personas con obesidad y sobrepeso no la realizan o lo hacen de manera ocasional ( $20,4 \%$ y $39,7 \%$ respectivamente).

El análisis del riesgo de presentar obesidad y sobrepeso frente al normopeso, según el consumo de diferentes grupos de alimentos, alcohol (cerveza y vino) y la actividad física durante el ocio se presenta en la Tabla 3. Atendiendo a las respuestas de los encuestados, la recomendación de la ingesta diaria de fruta se asoció con un mayor riesgo de presentar sobrepeso frente a la semanal (RRR ajustada $=0,77$; $p=, 008)$ y ocasional (RRR ajustada $=0,87 ; p=, 025)$. En cambio, la recomendación de la toma diaria de pasta-arroz (RRR ajustada $=1,37 ; p=, 037)$ y pan-cereales (RRR ajustada $=1,45$; $p=, 007)$ se relacionó con una menor probabilidad de tener sobrepeso frente a la ingesta semanal de ambos alimentos.

Tabla 3. Regresión logística multinomial del sobrepeso y la obesidad según consumo de alimentos, alcohol y actividad física en momentos de ocio. RRR crudas y ajustadas según variables sociodemográficas.

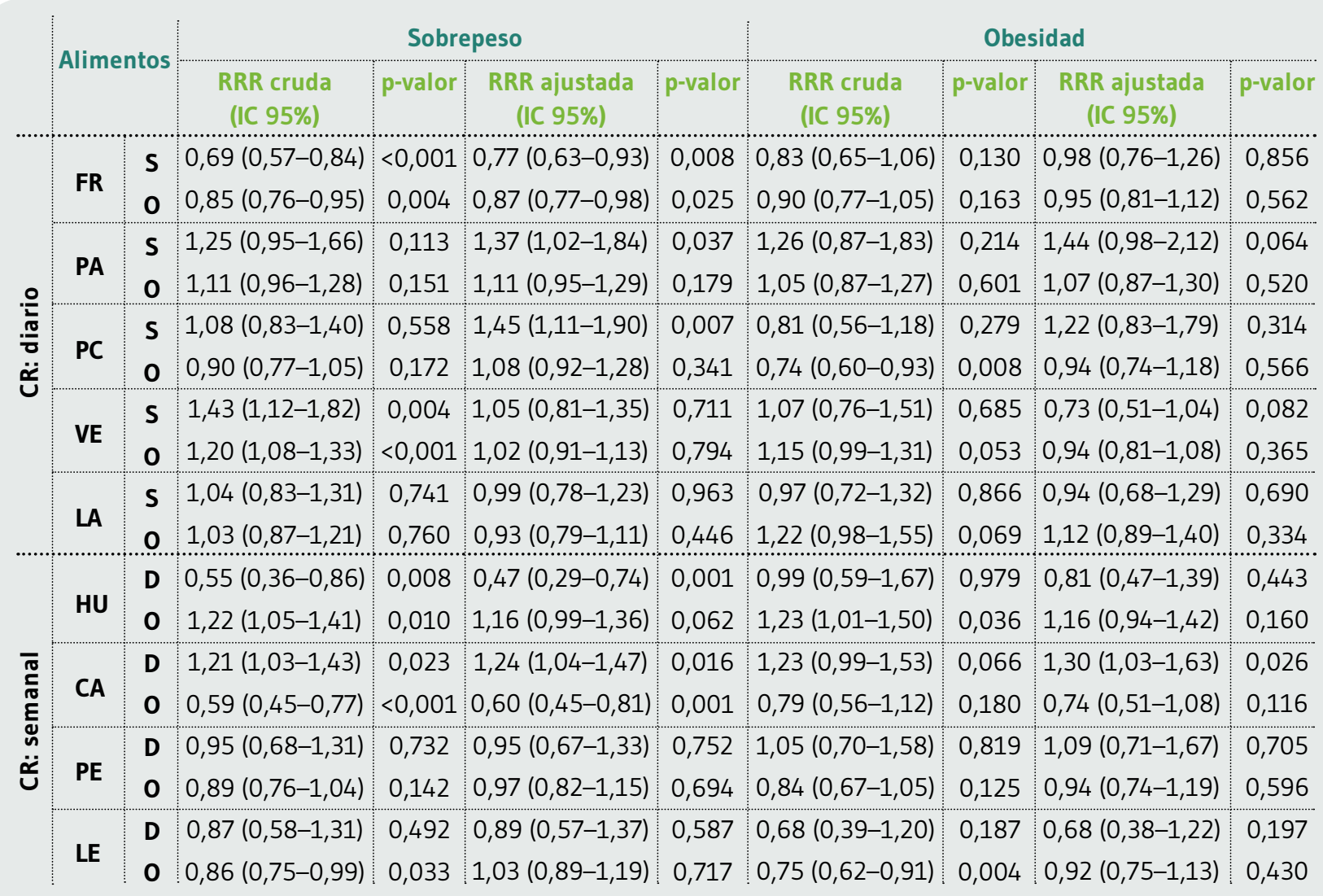




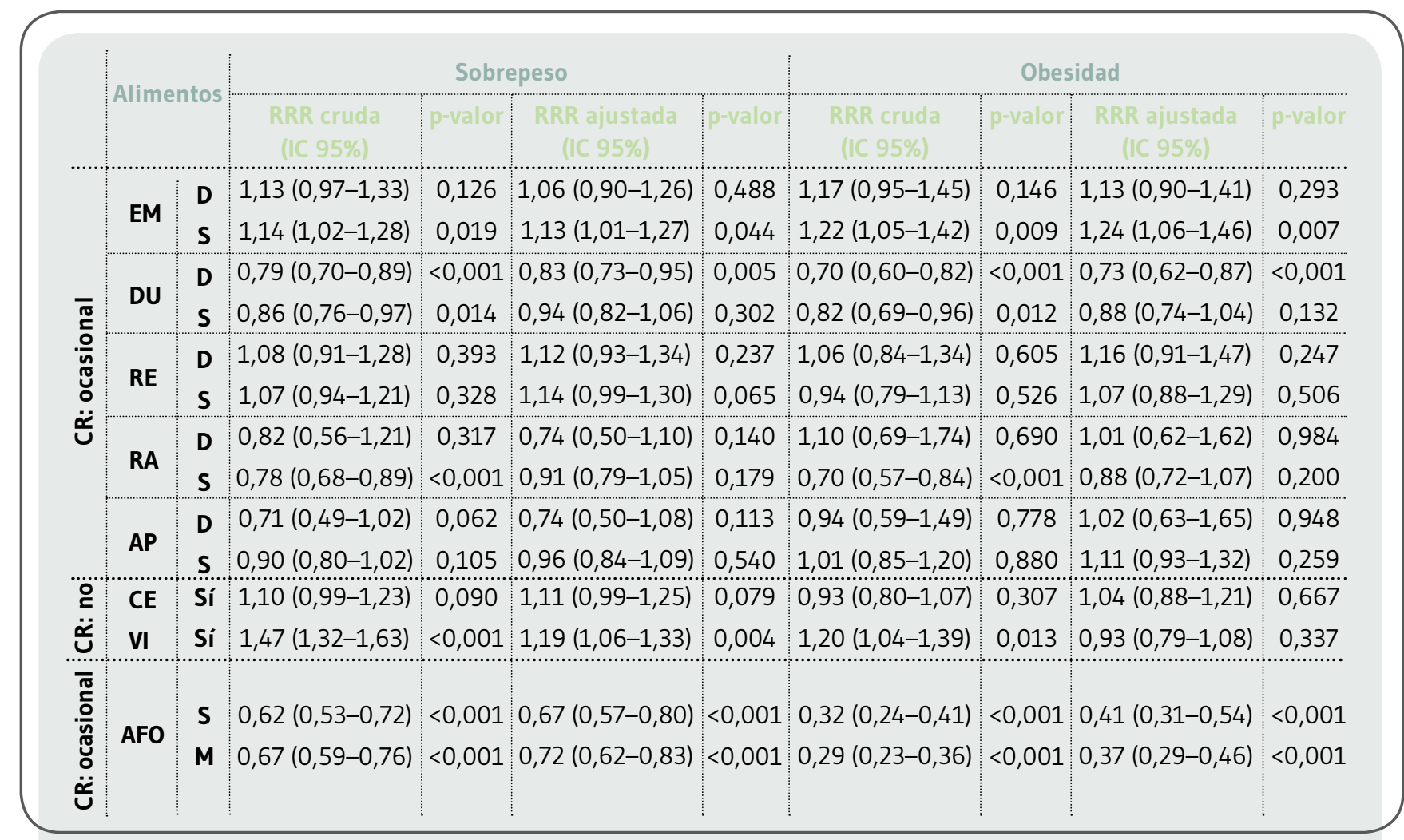

CR: categoría de referencia.

FR: fruta; PA: pasta y arroz; PC: pan y cereales; VE: verduras; LA: lácteos; HU: huevos; CA: carne; PE: pescado;

LE: legumbres; EM: embutidos; DU: dulces; RE: refrescos; RA: comida rápida; AP: aperitivos; CE: cerveza;

VI: vino; AFO: actividad física durante el ocio.

D: diario; S: semanal; 0: ocasional; M: mensual.

RRR ajustada a las siguientes variables sociodemográficas: edad, sexo, estado civil, tamaño del municipio, clase social, comunidad autónoma.

El consumo recomendado de huevos (semanal) se asoció a un mayor riesgo de padecer sobrepeso frente a la toma diaria de los mismos ( $R R R$ ajustada $=0,47 ; p=, 001$ ), mientras que la ingesta semanal de carne frente a la diaria se relacionó con una menor posibilidad de tener sobrepeso (RRR ajustada=1,24; $p=, 016)$, y de obesidad (RRR ajustada=1,30; $p=, 026)$. Respecto a los embutidos, su consumo ocasional frente al semanal se asoció con un menor riesgo de presentar sobrepeso (RRR ajustada $=1,13 ; p=, 044)$ y obesidad (RRR ajustada=1,24; $p=, 007)$. La ingesta ocasional de dulces frente a la diaria se relacionó con una mayor probabilidad de tener sobrepeso (RRR ajustada $=0,83 ; p=, 005)$ y de obesidad (RRR ajustada=0,73; $p<, 001$ ). En cuanto al alcohol, beber vino se relacionó con un aumento del riesgo de presentar sobrepeso (RRR ajustada=1,19; $p=, 004$ ). Todos los tamaños de efecto encontrados fueron muy pequeños. Los resultados obtenidos en cuanto a la actividad física durante el tiempo libre fueron más concluyentes ya que se obtuvieron tama- ños de efecto medianos. Realizar actividad física varias veces en semana o mensualmente disminuye significativamente el riesgo de padecer obesidad ( $R R R$ ajustada $=0,37 ; p<, 001$ ) y sobrepeso (RRR ajustada $=0,67 ; p<, 001)$.

\section{Discusión}

El presente estudio muestra que el consumo diario de fruta se asoció a un mayor riesgo de presentar sobrepeso y el consumo ocasional de dulces frente al diario se asoció a un mayor riesgo de tener sobrepeso y obesidad. Por otro lado, menos de la mitad de la población española siguió las recomendaciones de la SENC en cuanto al consumo de pasta y arroz, verduras, embutidos y dulces, y el $81,4 \%$ de los encuestados refirió no hacer actividad física nunca o casi nunca durante su tiempo de ocio. 
Según la $\mathrm{OMS}^{1}$, entre las principales causas de la actual pandemia de obesidad, se encuentran las dietas con alto contenido en grasas y el estilo de vida sedentario. Durante mucho tiempo se ha defendido el papel de la dieta mediterránea como protectora frente al desarrollo de sobrepeso y obesidad y otras enfermedades crónicas ${ }^{13,14}$ pero España cada vez se aleja más de esta dieta ${ }^{15}$. La dieta Mediterránea fue declarada Patrimonio Cultural Inmaterial de la Humanidad por la UNESCO en el año 2010 y las principales características de la misma son el elevado consumo de productos vegetales (frutas, verduras o legumbres), pan y cereales, el uso regular del aceite de oliva y un consumo moderado de vino en las comidas. Según resultados obtenidos, la población española presenta un déficit importante sobre todo en lo relacionado con el consumo de fruta y verdura.

La relación de los hábitos alimentarios con el sexo, muestra que las mujeres siguieron las recomendaciones de la SENC de un mayor grupo de alimentos que los hombres (fruta, verdura, lácteos, pescado, embutidos, refrescos, comida rápida y aperitivos). En esta línea, Arroyo y Cols. ${ }^{16}$, en un estudio de 2006 analizaron la calidad de la adherencia a las recomendaciones nutricionales y de actividad física separando los resultados por sexo, y mostraron que las mujeres obtuvieron puntuaciones más altas en el análisis del índice de calidad de la dieta, sobre todo en lo referente al consumo de frutas y verduras, mientras que los varones lo hicieron en cuanto a la actividad física. En 2011, se publicó otro trabajo en el que concluyeron que los hombres realizaban más actividad física que las mujeres ${ }^{17}$.

Los resultados obtenidos en cuanto a la asociación de los hábitos alimentarios con la presencia de sobrepeso y obesidad son contradictorios y similares a los encontrados por Norte Navarro y Cols. en $2006^{18}$, ya que muestran que el consumo diario de fruta se asoció a un mayor riesgo de sobrepeso, frente a la ingesta semanal y ocasional de la misma, y que la ingesta ocasional de dulces se asoció a una mayor probabilidad de sobrepeso y obesidad frente a la toma diaria de los mismos. Esto puede ser debido, en parte, a la limitación que tienen los estudios transversales, como es la ENS, para mostrar causalidad. En 2011, se publicó un consenso sobre las recomendaciones nutricionales basadas en la evidencia para la prevención y el tratamiento del sobrepeso y la obesidad en adultos ${ }^{19}$, en el que aparecía como controvertido que la toma de azúcares o aperitivos estuviera relacionada con el aumento de peso. Sin embargo, en 2015 se publicó un trabajo en British Journal of Nutrition en el que se relacionó el consumo de ciertos tipos de aperitivos y dulces con la presencia de obesidad ${ }^{20}$. En 2007, Schröder y Cols. publicaron un artículo en el que se hacía referencia a la asociación entre el consumo de comida rápida y la obesidad ${ }^{21} y$, sin embargo, Mesas y Cols. publicaron otro artículo en 2012 en el que expresaban la dificultad en la medición de estos hábitos alimentarios y que la ingesta de comida rápida, aperitivos, comer fuera de casa y otros hábitos que tradicionalmente se habían asociado con la presencia de sobrepeso y obesidad no estaban claramente relacionados con los mismos ${ }^{22}$.

El presente estudio muestra que la ingesta de vino se asocia a un mayor riesgo de sobrepeso, sin embargo esta asociación no está clara en la literatura ${ }^{23}$ y serían necesarios más estudios para valorar esta asociación.

La relación entre sedentarismo y obesidad está ampliamente documentada ${ }^{24}$. Los resultados obtenidos en el presente trabajo, referentes a la actividad física realizada por la población española, muestran que más de la mitad de los encuestados no la realiza nunca en su tiempo de ocio o lo hace de manera ocasional. Según un documento publicado por la OMS con respecto a las recomendaciones mundiales sobre actividad física para la salud ${ }^{25}$, los adultos deberían acumular un mínimo de 150 minutos semanales de actividad física aeróbica moderada, o bien 75 minutos de actividad física aeróbica vigorosa cada semana. La SENC recomienda la realización de una hora de actividad física al día ${ }^{11}$. Comparando estos datos con los obtenidos en el presente trabajo, observamos que se encuentran muy alejados de lo recomendado y quizá pueda influir en la elevada prevalencia de exceso de peso en nuestro país.

Los resultados de este estudio tienen una serie de limitaciones. Para el análisis de los hábitos alimentarios en la población española se han empleado varios métodos de evaluación dietética, directos e indirectos, y dentro de los primeros, los cuestionarios de frecuencia de consumo de alimentos (CFCA) han sido los más empleados. Los CFCA recogen principalmente datos sobre lista de alimentos, frecuencia y tamaño de la ración consumida. En aquellos casos en los que sólo se tienen en cuenta la lista de alimentos y su frecuencia, se denominan estudios cualitativos ${ }^{26}$ y la ENS se encuadraría en este grupo, siendo menos completos que los cuantitativos. De esta forma, la ENS no ofrece información acerca del tamaño de las raciones consumidas ni del número de raciones al día, por lo que los datos obtenidos pueden estar por encima o por debajo de lo recomendado sin poder diferenciarlo. Para que los resultados obtenidos de los CFCA sean fiables, deben ser validados ${ }^{27}$, algo que no ocurre con el CFCA de la ENS, por lo que los datos obtenidos pueden no mostrar asociaciones correctas al infraestimar el consumo ${ }^{28}$. Por otro lado, la ENS es un estudio transversal y, por lo tanto, no permite saber la direccionalidad de la asociación entre la alimentación, la realización de actividad física y la aparición de sobrepeso y obesidad. Además, es probable que las personas con obesidad sigan algún tipo de dieta para adelgazar y esto hace que sus hábitos de 
consumo, en un momento puntual, se puedan adecuar más a lo recomendado que si fueran tomados en períodos de tiempo mayores. En lo referente a la actividad física, la ENS no permite obtener datos válidos referentes al número de minutos empleados en la realización de la misma debido a la falta de respuestas de los encuestados, por lo que la comparación con las recomendaciones dadas por la OMS o la SENC es aproximada. Por otro lado, las recomendaciones sobre actividad física que hacen las Consejerías de Salud de los Gobiernos Autónomos, no se adaptan a las de la OMS y esto hace difícil poder realizar comparaciones entre los resultados obtenidos 29 .

\section{CONCLUSIONES}

La población española realiza poca actividad física. El consumo de pan y cereales, lácteos, huevos, carne, pescado y legumbres se adecua a las recomendaciones dadas por la SENC. Sin embargo, menos de la mitad de la población sigue las recomendaciones en cuanto al consumo de pasta y arroz, verduras, embutidos y dulces. Las mujeres siguen de forma significativa las recomendaciones de la mayor parte de los grupos de alimentos. El consumo diario de fruta y el consumo ocasional de dulces frente al consumo diario, se asocia a un mayor riesgo de sobrepeso y obesidad. La realización de actividad física varias veces en semana o mensualmente disminuye significativamente el riesgo de padecer sobrepeso y obesidad. Este trabajo muestra la relación entre el exceso de peso y determinados hábitos alimentarios y patrones de ejercicio físico. El adecuado conocimiento de los mismos puede ayudar a la elaboración de un plan preventivo a nivel nacional que permita frenar el crecimiento del sobrepeso y la obesidad en nuestro país.

\section{$\longrightarrow$ \\ CONFLICTO DE INTERESES}

Los autores expresan que no hay conflictos de interés al redactar el manuscrito.

\section{FINANCIACIÓN}

Este estudio fue financiado parcialmente por un proyecto de investigación concedido por la Fundación Universidad Alfonso X el Sabio y el Grupo Santander en el año 2015.

\section{REFERENCIAS}

(1) Bessesen DH. Update on obesity. J Clin Endocrinol Metab. 2008; 93(6): 2027-34.

(2) Organización Mundial de la Salud. OMS | Obesidad y sobrepeso - Nota descriptiva N³11 [Internet]. WHO. 2016 [citado 1 de febrero de 2016]. Disponible en: http://www.who.int/ mediacentre/factsheets/fs311/es/

(3) Robertson A, Lobstein T, Knai C. Obesity and socio-economic groups in Europe: Evidence review and implications for action. Bruselas, Bélgica: European Commission; 2007.

(4) Gutiérrez-Fisac JL, Regidor E, Banegas JR, Rodríguez Artalejo F. Prevalencia de Obesidad en la población adulta española: 14 años de incremento continuado. Med Clin. 2005; 124(5): 196-7.

(5) AESAN. ENIDE - Encuesta Nacional de Ingesta Dietética Española 2011. 2011; Madrid, España.

(6) Aranceta-Bartrina J, Varela-Moreiras G, Serra-Majem L, PérezRodrigo C, Abellana R, Ara I, et al. Consensus document and conclusions. Methodology of dietary surveys, studies on nutrition, physical activity and other lifestyles. Nutr Hosp. 2015; 31(Suppl 3): 9-12.

(7) Ruiz E, Ávila JM, Valero T, del Pozo S, Rodriguez P, ArancetaBartrina J, et al. Energy Intake, Profile, and Dietary Sources in the Spanish Population: Findings of the ANIBES Study. Nutrients. 2015; 7(6): 4739-62.

(8) Varela-Moreiras G, Alguacil Merino LF, Alonso Aperte E, Aranceta Bartrina J, Avila Torres JM, Aznar Laín S, et al. CONSENSUS DOCUMENT AND CONCLUSIONS - Obesity and sedentarism in the 21st century: what can be done and what must be done? Nutr Hosp. 2013; 28(Suppl 5): 1-12.

(9) Ministerio de Sanidad, Servicios Sociales e Igualdad. Encuesta Nacional de Salud de España 2011/2012 [Internet]. Ministerio de Sanidad, Servicios Sociales e Igualdad. 2013 [citado 1 de octubre de 2015]. Disponible en: https://www.msssi.gob.es/ estadEstudios/estadisticas/encuestaNacional/encuesta2011. htm

(10) World Health Organization. Obesity: preventing and managing the global epidemic. Report of a WHO consultation. World Health Organ Tech Rep Ser. 2000; 894.

(11) Sociedad Española de Nutrición Comunitaria, Sociedad Española de Medicina de Familia y Comunitaria, editores. Consejos para una alimentación saludable. Madrid, España: SENC y semFYC; 2007.

(12) Marqueta de Salas M, Martín-Ramiro Jj, Juárez Soto J]. Características sociodemográficas como factores de riesgo para la obesidad y el sobrepeso en la población adulta española. Med Clin. 2016; 146(11): 471-7.

(13) Beunza J-J, Toledo E, Hu FB, Bes-Rastrollo M, Serrano-Martínez $M$, Sánchez-Villegas $A$, et al. Adherence to the Mediterranean diet, long-term weight change, and incident overweight or obesity: the Seguimiento Universidad de Navarra (SUN) cohort. Am J Clin Nutr. 2010; 92(6): 1484-93. 
(14) Fundación Española de la Nutrición, editor. Libro blanco de la nutrición en España. Madrid: Fundación Española de la Nutrición; 2013.

(15) Bach-Faig A, Fuentes-Bol C, Ramos D, Carrasco JL, Roman B, Bertomeu IF, et al. The Mediterranean diet in Spain: adherence trends during the past two decades using the Mediterranean Adequacy Index. Public Health Nutr. 2011; 14(4): 622-8.

(16) Arroyo Izaga M, Rocandio Pablo AM, Ansotegui Alday L, Pascual Apalauza E, Salces Beti I, Rebato Ochoa E. Calidad de la dieta, sobrepeso y obesidad en estudiantes universitarios. Nutr Hosp. 2006; 21(6): 673-9.

(17) Arbinaga F, García D, Vázquez I, Joaquín M, Pazos E. Actitudes hacia el ejercicio en estudiantes universitarios: relaciones con los hábitos alimenticios y la insatisfacción corporal. Rev Iber Psic Ejerc Dep. 2011; 6(1): 97-112.

(18) Norte-Navarro Al, Ortiz-Moncada R, Fernández-Sáez J, ÁlvarezDardet C. Asociación entre la dieta y la presencia de sobrepeso y obesidad. Método de evaluación dietética de la Encuesta Nacional de Salud Española 2006. Rev Esp Nutr Hum Diet. 2013; 17(3): 102-9.

(19) Gargallo M, Basulto J, Breton I, Quiles J, Formiguera X, SalasSalvadó J, et al. FESNAD-SEEDO consensus summary: Evidencebased nutritional recommendations for the prevention and treatment of overweight and obesity in adults. Endocrinol Nutr. 2012; 59(7): 429-37.

(20) O'Connor L, Brage S, Griffin SJ, Wareham NJ, Forouhi NG. The cross-sectional association between snacking behaviour and measures of adiposity: the Fenland Study, UK. Br ] Nutr. 2015; 114(8): 1286-93.
(21) Schröder H, Fïto M, Covas MI, REGICOR investigators. Association of fast food consumption with energy intake, diet quality, body mass index and the risk of obesity in a representative Mediterranean population. Br J Nutr. 2007; 98(6): 1274-80.

(22) Mesas AE, Muñoz-Pareja M, López-García E, Rodríguez-Artalejo F. Selected eating behaviours and excess body weight: a systematic review. Obes Rev. 2012; 13(2): 106-35.

(23) Pimentel GD, Lira FS, Rosa JC. El consumo de alcohol y la desregulación hormonal de la ingesta de alimentos: sería un camino a la contribución de la obesidad? Nutr Hosp. 2011; 26(4): 926.

(24) Fox KR, Hillsdon M. Physical activity and obesity. Obes Rev. 2007; 8(Suppl 1): 115-21.

(25) Organización Mundial de la Salud. Recomendaciones mundiales sobre actividad física para la salud. Ginebra, Suiza: Organización Mundial de la Salud; 2010.

(26) Pérez C, Aranceta J, Salvador G, Varela-Moreiras G. Food frequency questionnaires. Nutr Hosp. 2015; 31(Suppl 3): 49-56.

(27) Castro Rodríguez P, Bellido Guerrero D, Pertega Díaz S, Grupo Colaborativo del Estudio. Elaboración y validación de un nuevo cuestionario de hábitos alimentarios para pacientes con sobrepeso y obesidad. Endocrinol Nutr. 2010; 57(4): 130-9.

(28) Molag ML, de Vries JHM, Ocké MC, Dagnelie PC, van den Brandt $\mathrm{PA}$, Jansen MCJF, et al. Design characteristics of food frequency questionnaires in relation to their validity. Am J Epidemiol. 2007; 166(12): 1468-78.

(29) Chodzko-Zajko WJ, Schwingel A, Romo-Pérez V. Un análisis crítico sobre las recomendaciones de actividad física en España. Gac Sanit. 2012; 26(6): 525-33. 